\title{
Physiological Evaluation of Genotypes for Drought Tolerance on Receding Soil Moisture
}

\author{
U.D. Chavan", S.V. Nirmal, S.R. Gadakh, M.S. Shinde, \\ V.R. Patil, V.R. Awari and U.S. Dalvi
}

All India Coordinated Sorghum Improvement Project, Mahatma Phule Krishi

Vidyapeeth, Rahuri, India

*Corresponding author

A B S T R A C T

Keywords

Sorghum genotypes, physiological characteristics, RLWC, SPAD value, Medium soil, Harvest index, Leaf temperature.

Article Info

Accepted:

26 May 2017

Available Online:

10 June 2017
Thirteen new Rabi sorghum genotypes and three checks were evaluated for their physiological characterization under medium and shallow soils during Rabi season viz., 2014-15, 2015-16 and 2016-17 in randomized block design with three replications. The grain yield was positively correlated with leaf area, leaf area index, biomass at harvest, HI \%, 1000 grain weight, RLWC \%, stay green at physiological maturity, while per day production of grain and fodder yield negatively correlated with leaf temperature differences. While considering all physiological parameters on medium soil genotype RSSV 1640 was found significantly superior over the checks. On shallow soil, none of the new genotype was found significantly superior over the check Phule Anuradha.

\section{Introduction}

Sorghum (Sorghum bicolor L. Moench) is the king of cereals and is one of the important food crops in dry lands of tropical Africa, India and China (Shobha et al., 2008). India ranks second in the world for sorghum production and first with respect to many regionally important crops like millets and pseudo-cereals. Sorghum is the principal staple food of Maharashtra, and is also an important food of Karnataka, Madhya Pradesh, Tamil Nadu and Andhra Pradesh.
Sorghum can be milled to produce starch or grits (semolina) from which many ethnic and traditional dishes can be made. The most common products are leavened and unleavened breads, porridges, boiled grains and steam cooked products such as couscous. Sorghum flour also makes an excellent fry coating for fish, chicken and beef. Sorghum is also used in the preparation of several snacks and for popping, chewing, and malting (Rao and Murty, 1981). There is a considerable 
variation in sorghum for levels of proteins, lysine, lipids, carbohydrates, fiber, calcium, phosphorus, iron, thiamine, and niacin (Chavan et al., 2009). Sorghum has chemical composition similar to or better than rice and wheat in some respects. The grains contain high fiber and non-starchy polysaccharides and starch with some unique characteristics. Protein quality and essential amino acid profile of sorghum is better than many of the cereals. Sorghum in general is rich source of B-complex vitamins (Chavan et al., 1988; 2010; 2015).

Sorghum roti is very popular in villages and small towns as an accompaniment to gravy meat and vegetable curries and is one of the traditional recipes of India. It is round, flat; unleavened bread often used in the cuisine of western and central India, especially in the states of Gujarat, Sorghum roti is known by various names in the different languages of India: chapati (Hindi), bhakri (Marathi), rotla (Gujarati), rotte (Telugu), etc. (Subramanian and Jambunathan, 1981). Because sorghum flour is gluten-free flour, it is very tough to spread the dough without breaking the shape and one really needs hands-on experience and many failed attempts to get the skill. No leavening agents, oil/ghee are added. Just fresh sorghum flour, warm water and touch of fire - pure grain power in its glory. Arabinoxylans have been isolated from different cereals and responsible to play important role in maintaining water balance and rheological properties of dough (Michniewicz et al., 1991; Vietor et al., 1992; Nandini et al., 2001).

Typically bhakri is accompanied by various curries, chutney (thecha - a thick paste of really hot green or red chilies) and raw onion (Murty and Subramanian, 1981). Bhakri has its own advantages from dietary point of view. Being made from cereals, it is high in dietary fiber but at the same time very easy to digest (Chavan et al., 1989, Chavan and Patil, 2010 and Chavan and Salunkhe, 1984). However it was necessary to study the effect different locations on the nutritional and roti quality therefore, present study was under taken to identify superior genotype for future development.

\section{Materials and Methods}

Thirteen new Rabi sorghum genotypes and three checks were evaluated for their physiological characterization under medium and shallow soils at Sorghum Improvement Project, Mahatma Phule Krishi Vidyapeeth, Rahuri during Rabi season viz., 2014-15, 2015-16 and 2016-17 in randomized block design with three replications. The gross and net plot size was $4.50 \times 2.70 \mathrm{~m}$ and $4.10 \mathrm{x}$ $1.80 \mathrm{~m}$, respectively and the sowing was done by keeping $45 \quad \mathrm{x} \quad 15 \mathrm{~cm}$ spacing. All agronomic practices were followed as a when required. Goth and physiological parameters were recorded at $50 \%$ flowering and grain yield data was recorded after the harvest of the crop.

\section{Plant height (cm)}

Plant height of five randomly selected plants from each plot was recorded in $\mathrm{cm}$ by measuring from the base of the plant near the ground up to the tip of the panicle.

\section{Leaf area $\left(\mathrm{dm}^{2}\right)$}

The leaf area of the plant was calculated by taking maximum length and width at the broadest point of the green leaves and multiplying by the factor 0.747 (Stickler et al., 1961).

\section{Leaf area index (LAI)}

From the data of leaf area per plant the leaf index was calculated according to the 
following formula by Watson (1947).

LAI $=\frac{\text { Leaf area per plant }\left(\mathrm{dm}^{2}\right)}{\text { Land area }\left(\mathrm{dm}^{2}\right)} \quad$ PA

\section{Relative leaf water content (RLWC, \%)}

The relative leaf water content was determined according to the procedure given by Barrs and Weatherly (1962) at flowing stage and it was expressed in percent $(\%)$.

Fresh weight $(\mathrm{Wf})-$ Dry weight $(\mathrm{Wd})$ $\operatorname{RLWC}(\%)=$ X 100

Turgid weight $(\mathrm{Wt})-$ Dry weight $(\mathrm{Wd})$

\section{Dry matter (\%)}

The dry matter at 50\% flowering, of leaf, stem and panicle were recorded using standard methods.

\section{Heat unit}

The total heat units required from sowing to panicle ignition, panicle flowing, flowering to physiological maturity worked by the total submission of the degree days above the base temperature with the help of formula suggested by Iwata (1984) Base temperature taken as $10{ }^{\circ} \mathrm{C}$ (Klages, 1958).

Heat unit $=\sum_{i=1}^{n}$ - Max tem. + Min. tem.

\section{Heat unit efficiency (HUF)}

The heat unit efficiency calculated for grain and dry matter per plant at maturity by the formula given by Rajput (1980).

Heat unit efficiency

$$
\text { Grain yield }(\mathrm{g})
$$

Total heat unit (sowing to maturity)

Heat unit efficiency

Dry matter $(\mathrm{g})$

(Dry matter yield $)=$

Total heat unit (sowing to maturity)

\section{Thousand grain weight (g)}

Thousand grains of each genotype were counted and weight (g) was recorded by electronic balance.

\section{Harvest index (\%)}

The harvest index (HI) expressed in (\%) was calculated by using the formula given by Donald (1962).

Economic yield (g/plant)

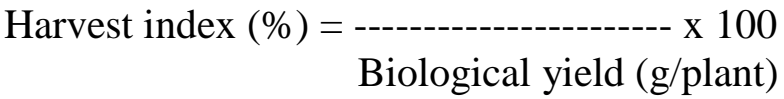

\section{Other parameters}

The standard methods given in the AOAC (1990) were used to record the observations and yield of the crop.

\section{Statistical analysis}

The statistical analysis of the data was carried out by Analysis of variance method suggested by Panse and Sukhatme (1985).

\section{Results and Discussion}

Sorghum crop is included in the C-4 plants which have high capacity of photosynthesis. The photosynthesis is mostly depends on the leaf area of that plant. The leaf area and leaf area index showed positive correlation to yield parameters (Table 1a). The genotype RSV 1640 gave highest leaf area and leaf area index which governs the final yield of the crop for medium soil type. But for shallow 
soil type Phule Anuradha showed higher level of yield. LAI helps in intercepting more solar radiation and thereby enhancing photosynthetic rate and resulting in higher total dry matter production and grain yield.

Leaf area index is an important parameter which governs dry matter production and thus yields (Kulkarni et al., 1981).

Similar findings were also reported by Haremath and Parvatikar (1985).

The genotype RSV 1640 recorded higher RLWC (90.00\%) as compared with all checks in medium soil (Table 1a).

But for shallow soil the check genotype Phule Anuradha showed lowest RLWC than other genotypes studied (Table 2a). Nirmal et al., (2013) reported that the RLWC \% under rainfed condition and irrigated conditions ranged from $49-76 \%$ and $48-76 \%$ respectively.

The SPAD value of RSV 1640 was medium but their leaf temperature difference was highest (-7.1) as compared to other all genotypes studied in medium soil type.

This indicated that more energy is used for the development of yield component during their growth development. In shallow soil Phule Anuradha showed highest SPAD value than other genotypes studied (Table 2a). Similar results are also reported by several researchers.

The heat unit and heat unit efficiency also play important role in the directly utilization energy for growth and yield components. The new genotype RSV 1640 showed 89\% energy efficiency in medium soil (Table 1a).

In shallow soil also Phule Anuradha check genotype showed $74 \%$ efficiency in the utilization of heat energy in to yield production in Rabi sorghum (Table 2a). Similar results also reported by Nirmal et al., (2013).

Shinde and Narkhede (1998) studied the heat unit required of parental lines during kharif, rabi and summer season. The results revealed that heat units were lower in rabi season and higher in summer flowed by kharif.

For yield contributing characters it was indicated that highest biomass at flowering was produced by the genotype BRJ 341 (6395 $\mathrm{kg} / \mathrm{ha})$ and at harvest $9280(\mathrm{~kg} / \mathrm{ha})$ on medium soil and the BRJ 235 produced highest biomass at flowering $(287(\mathrm{~kg} / \mathrm{ha})$ and at harvest $4776(\mathrm{~kg} / \mathrm{ha})$ on shallow soil (Table 2 $a, b)$.

As regards per day production ( $\mathrm{kg} / \mathrm{ha} /$ day) the genotype RSV 1640 produced higher grain 28.35 (kg/ha/day) and fodder 42.47 ( $\mathrm{kg} / \mathrm{ha} /$ day) on medium soil and genotype Phule Anuradha produced higher grains (8.20 ( $\mathrm{kg} / \mathrm{ha} /$ day) and fodder 22.70 ( $\mathrm{kg} / \mathrm{ha} /$ day) on shallow soil (Table $1 \mathrm{~b}$ and 2b). Kusalkar et al., (2003) also reported similar results.

The dry matter yield at harvest is mostly based on the harvest index. For medium soil type genotype RSV 1640 showed highest harvest index (40) than other new genotypes, while for shallow soil type the genotype Phule Anuradha showed highest harvest index 27 (Table 2a).

The genotype Phule Suchitra produced higher 1000-seed weight (40/1000 grains) on medium soil and BRJ 341 and Phule Suchitra gave highest 100-seed weight (34/1000 grains) on shallow soil. These genotypes also showed 5-6 range for the stay green at physiological maturity stage on both medium and shallow soil (Table $1 \mathrm{~b}$ and $2 \mathrm{~b}$ ). Narkhede et al., (1998) and Nirmal and Patil (2008) also reported similar results. 
Table.1a Physiological traits associated with grain and fodder yield (Medium soil)

\begin{tabular}{|c|c|c|c|c|c|c|c|c|c|c|c|c|}
\hline \multirow[t]{2}{*}{ Entry } & \multirow{2}{*}{$\begin{array}{l}\text { Leaf } \\
\text { area } \\
\left(\mathrm{dm}^{2}\right)\end{array}$} & \multirow[t]{2}{*}{ LAI } & \multirow{2}{*}{$\begin{array}{l}\text { RLWC } \\
(\%)\end{array}$} & \multirow{2}{*}{$\begin{array}{l}\text { SPAD } \\
\text { at } \\
\text { flow. }\end{array}$} & \multirow{2}{*}{$\begin{array}{l}\text { Leaf } \\
\text { temp. } \\
\text { Diff. } \\
\left({ }^{0} \mathrm{C}\right)\end{array}$} & \multirow{2}{*}{$\begin{array}{l}\text { Heat } \\
\text { Unit }\end{array}$} & \multirow{2}{*}{$\begin{array}{l}\text { Heat } \\
\text { Unit } \\
\text { Efficie } \\
\text { ncy }\end{array}$} & \multirow[t]{2}{*}{$\mathrm{EE}(\%)$} & \multirow{2}{*}{$\begin{array}{l}\text { Biologic } \\
\text { al Yield } \\
\mathrm{kg} / \mathrm{ha}\end{array}$} & \multirow[t]{2}{*}{$\mathrm{HI}$} & \multicolumn{2}{|c|}{ Yield (kg ha-1) } \\
\hline & & & & & & & & & & & Grain & Fodder \\
\hline RSV1544 & 33.8 & 5.02 & 86 & 54.9 & -6.7 & 1395 & 2.209 & 85 & 8731 & 35 & 3082 & 5649 \\
\hline RSV1572 & 25.7 & 3.81 & 90 & 58.2 & -6.2 & 1395 & 1.928 & 69 & 6804 & 40 & 2690 & 4114 \\
\hline RSV1620 & 29.9 & 4.43 & 86 & 63.6 & -6.2 & 1435 & 2.291 & 77 & 9518 & 35 & 3287 & 6231 \\
\hline RSV1640 & 34.6 & 5.11 & 90 & 56.9 & $\begin{array}{l}-7.1 \\
\end{array}$ & 1413 & 2.388 & 89 & 8428 & 40 & 3374 & 5054 \\
\hline RSSV167 & 30.6 & 4.54 & 88 & 53.7 & -5.2 & 1484 & 2.123 & 81 & 10467 & 30 & 3150 & 7317 \\
\hline CRS 49 & 24.0 & 3.57 & 86 & 54.2 & -6.1 & 1413 & 1.778 & 83 & 8396 & 30 & 2513 & 5883 \\
\hline CRS 50 & 29.9 & 4.43 & 85 & 55.4 & -6.6 & 1484 & 1.811 & 72 & 9645 & 28 & 2687 & 6958 \\
\hline PVR 930 & 29.9 & 4.43 & 83 & 52.9 & -6.0 & 1421 & 2.082 & 77 & 7732 & 38 & 2958 & 4774 \\
\hline BRJ 229 & 33.8 & 5.02 & 87 & 53.0 & -6.4 & 1484 & 1.953 & 79 & 8807 & 33 & 2898 & 5909 \\
\hline BRJ 343 & 34.6 & 5.11 & 86 & 53.6 & -5.5 & 1472 & 2.163 & 83 & 9508 & 33 & 3184 & 6324 \\
\hline BRJ 235 & 25.4 & 3.76 & 86 & 54.7 & -7.1 & 1489 & 2.074 & 74 & 8915 & 35 & 3088 & 5827 \\
\hline BRJ 376 & 28.8 & 4.24 & 75 & 52.5 & -6.5 & 1472 & 1.708 & 82 & 9141 & 28 & 2514 & 6627 \\
\hline BRJ 341 & 26.4 & 3.92 & 86 & 54.7 & -6.3 & 1460 & 1.993 & 79 & 9280 & 31 & 2910 & 6370 \\
\hline M-35-1 (C) & 26.7 & 3.96 & 81 & 51.8 & -6.3 & 1441 & 1.986 & 85 & 7205 & 40 & 2862 & 4343 \\
\hline P. Suchitra (C) & 29.8 & 4.42 & 83 & 53.7 & -6.2 & 1472 & 2.213 & 76 & 8980 & 36 & 3258 & 5722 \\
\hline P. Anuradha (C) & 22.6 & 3.36 & 76 & 56.8 & -6.2 & 1403 & 1.964 & 81 & 6134 & 45 & 2755 & 3379 \\
\hline $\mathrm{SE} \pm$ & & & & & & & & & & & 126.8 & 271.3 \\
\hline $\mathrm{CD}$ at $5 \%$ & & & & & & & & & & & 366.1 & 783.4 \\
\hline $\mathrm{CV} \%$ & & & & & & & & & & & 7.44 & 8.31 \\
\hline Correlation & +0.38 & +0.26 & +0.32 & +0.48 & -0.67 & +0.38 & +0.45 & +0.18 & +0.45 & +0.53 & & +0.43 \\
\hline
\end{tabular}


Table.1b Physiological traits associated with grain and fodder yield (Medium soil)

\begin{tabular}{|c|c|c|c|c|c|c|c|c|c|c|c|}
\hline \multirow[t]{2}{*}{ Entry } & \multirow[t]{2}{*}{$\begin{array}{l}\text { Plant } \\
\text { height } \\
(\mathrm{cm})\end{array}$} & \multirow{2}{*}{$\begin{array}{l}\text { Biomass } \\
\text { at } \\
50 \\
\text { flow. } \\
(\mathrm{kg} / \mathrm{ha})\end{array}$} & \multirow{2}{*}{$\begin{array}{l}1000 \\
\text { grain } \\
\text { weight } \\
(\mathrm{g})\end{array}$} & \multicolumn{2}{|l|}{ Days to } & \multicolumn{2}{|c|}{$\begin{array}{l}\text { Per days } \\
\text { production } \\
\mathrm{kg} / \text { ha/day }\end{array}$} & \multicolumn{3}{|c|}{$\begin{array}{l}\text { Dry matter at } 50 \% \text { flow. } \\
\left(\mathrm{g} / \mathrm{m}^{2}\right)\end{array}$} & \multirow[t]{2}{*}{$\begin{array}{l}\text { Stay green at } \\
\text { phy. maturity } \\
(1-9 \text { scale })\end{array}$} \\
\hline & & & & $\begin{array}{l}50 \% \\
\text { flow. }\end{array}$ & $\begin{array}{l}\text { Phy. } \\
\text { maturity }\end{array}$ & Grain & Fodder & Leaves & Stem & Panicle & \\
\hline RSV1544 & 232 & 4888 & 33 & 75 & 115 & 26.80 & 49.12 & 79 & 253 & 64 & $5-6$ \\
\hline RSV1572 & 208 & 5419 & 32 & 75 & 115 & 23.39 & 35.77 & 78 & 300 & 61 & $5-6$ \\
\hline RSV1620 & 232 & 4864 & 32 & 80 & 123 & 26.72 & 50.66 & 78 & 256 & 60 & $5-6$ \\
\hline RSV1640 & 230 & 4321 & 32 & 77 & 119 & 28.35 & 42.47 & 80 & 209 & 61 & $5-6$ \\
\hline RSSV167 & 227 & 4851 & 36 & 85 & 126 & 25.00 & 58.07 & 79 & 261 & 52 & $5-6$ \\
\hline CRS 49 & 206 & 3975 & 37 & 77 & 115 & 21.85 & 51.16 & 77 & 189 & 56 & $5-6$ \\
\hline CRS 50 & 214 & 5370 & 38 & 85 & 126 & 21.33 & 55.22 & 97 & 272 & 65 & $7-8$ \\
\hline PVR 930 & 201 & 4160 & 33 & 77 & 118 & 25.07 & 40.46 & 81 & 189 & 68 & $5-6$ \\
\hline BRJ 229 & 226 & 5000 & 35 & 85 & 124 & 23.37 & 47.65 & 95 & 247 & 64 & $7-8$ \\
\hline BRJ 343 & 230 & 5543 & 33 & 84 & 124 & 25.68 & 51.00 & 99 & 283 & 67 & $7-8$ \\
\hline BRJ 235 & 209 & 5172 & 31 & 86 & 125 & 24.70 & 46.62 & 97 & 255 & 67 & $5-6$ \\
\hline BRJ 376 & 216 & 4185 & 32 & 84 & 124 & 20.27 & 53.44 & 92 & 185 & 62 & $7-8$ \\
\hline BRJ 341 & 224 & 6395 & 39 & 83 & 119 & 24.45 & 53.53 & 86 & 367 & 65 & $5-6$ \\
\hline M-35-1 (C) & 217 & 5555 & 35 & 81 & 120 & 23.85 & 36.19 & 81 & 319 & 49 & $7-8$ \\
\hline P. Suchitra (C) & 221 & 5666 & 40 & 84 & 125 & 26.06 & 45.78 & 94 & 298 & 67 & $5-6$ \\
\hline P. Anuradha (C) & 198 & 4358 & 35 & 76 & 108 & 24.17 & 29.64 & 73 & 222 & 58 & $5-6$ \\
\hline Correlation & +0.648 & +0.531 & +0.367 & +0.289 & +0.063 & +0.319 & +0.068 & +0.238 & +0.489 & +0.639 & - \\
\hline
\end{tabular}


Table.2a Physiological traits associated with grain and fodder yield (Shallow soil)

\begin{tabular}{|c|c|c|c|c|c|c|c|c|c|c|c|c|}
\hline \multirow[t]{2}{*}{ Entry } & \multirow{2}{*}{$\begin{array}{l}\text { Leaf } \\
\text { area } \\
\left(\mathrm{dm}^{2}\right)\end{array}$} & \multirow[t]{2}{*}{ LAI } & \multirow{2}{*}{$\begin{array}{l}\text { RLW } \\
\text { C (\%) }\end{array}$} & \multirow{2}{*}{$\begin{array}{l}\text { SPAD } \\
\text { at } \\
\text { flow. }\end{array}$} & \multirow{2}{*}{$\begin{array}{l}\text { Leaf } \\
\text { temp. } \\
\text { Diff. } \\
\left({ }^{0} \mathrm{C}\right)\end{array}$} & \multirow{2}{*}{$\begin{array}{l}\text { Heat } \\
\text { Unit }\end{array}$} & \multirow{2}{*}{$\begin{array}{l}\text { Heat } \\
\text { Unit } \\
\text { Efficie } \\
\text { ncy }\end{array}$} & \multirow{2}{*}{$\begin{array}{l}\mathrm{EE} \\
(\%)\end{array}$} & \multirow{2}{*}{$\begin{array}{l}\text { Biolo } \\
\text { gical } \\
\text { Yield } \\
\text { kg/ha }\end{array}$} & \multirow[t]{2}{*}{$\mathrm{HI}$} & \multicolumn{2}{|c|}{ Yield (kg ha-1) } \\
\hline & & & & & & & & & & & Grain & Fodder \\
\hline RSV1544 & 20.2 & 2.99 & 84 & 39.9 & -3.9 & 1385 & 0.675 & 75 & 4775 & 20 & 905 & 3840 \\
\hline RSV1572 & 20.2 & 2.99 & 89 & 44.2 & -3.9 & 1395 & 0.518 & 70 & 4756 & 15 & 723 & 4033 \\
\hline RSV1620 & 17.1 & 2.54 & 81 & 33.4 & -3.4 & 1413 & 0.585 & 52 & 4773 & 17 & 827 & 3946 \\
\hline RSV1640 & 17.8 & 2.57 & 89 & 40.1 & -3.4 & 1395 & 0.524 & 65 & 3101 & 24 & 731 & 2370 \\
\hline RSSV167 & 20.5 & 3.03 & 81 & 40.6 & -3.5 & 1429 & 0.624 & 51 & 3732 & 24 & 891 & 2841 \\
\hline CRS 49 & 14.3 & 2.13 & 79 & 40.8 & -3.8 & 1385 & 0.624 & 63 & 4456 & 19 & 864 & 3592 \\
\hline CRS 50 & 14.0 & 2.07 & 83 & 39.2 & -3.2 & 1435 & 0.594 & 55 & 3525 & 24 & 853 & 2672 \\
\hline PVR 930 & 15.3 & 2.27 & 77 & 34.6 & -2.8 & 1395 & 0.652 & 55 & 2928 & 31 & 909 & 2019 \\
\hline BRJ 229 & 18.7 & 2.78 & 82 & 38.9 & -2.7 & 1435 & 0.620 & 47 & 3179 & 28 & 889 & 2290 \\
\hline BRJ 343 & 17.6 & 2.60 & 81 & 44.1 & -3.9 & 1435 & 0.576 & 64 & 3453 & 24 & 827 & 2626 \\
\hline BRJ 235 & 13.2 & 1.96 & 84 & 41.5 & -3.3 & 1421 & 0.643 & 61 & 4776 & 19 & 914 & 3862 \\
\hline BRJ 376 & 15.4 & 2.27 & 77 & 39.0 & -3.4 & 1441 & 0.609 & 50 & 3559 & 25 & 878 & 2681 \\
\hline BRJ 341 & 15.6 & 2.31 & 81 & 35.2 & -2.8 & 1429 & 0.304 & 50 & 2812 & 15 & 434 & 2378 \\
\hline M-35-1 (C) & 17.1 & 2.53 & 81 & 42.2 & -3.4 & 1429 & 0.452 & 62 & 3774 & 17 & 646 & 3128 \\
\hline P. Suchitra (C) & 18.5 & 2.74 & 72 & 37.1 & -3.3 & 1435 & 0.484 & 59 & 3982 & 17 & 694 & 3288 \\
\hline P. Anuradha (C) & 18.9 & 2.78 & 68 & 44.4 & -4.0 & 1385 & 0.675 & 74 & 3523 & 27 & 935 & 2588 \\
\hline $\mathrm{SE} \pm$ & & & & & & & & & & & 61.60 & 96.00 \\
\hline $\mathrm{CD}$ at $5 \%$ & & & & & & & & & & & 177.9 & 277.3 \\
\hline $\mathrm{CV} \%$ & & & & & & & & & & & 13.18 & 5.52 \\
\hline Correlation & +0.89 & +0.36 & +0.37 & +0.67 & -0.29 & +0.349 & +0.272 & +0.72 & +0.09 & +0.27 & - & +0.25 \\
\hline
\end{tabular}


Table.2b Physiological traits associated with grain and fodder yield (Shallow soil)

\begin{tabular}{|c|c|c|c|c|c|c|c|c|c|c|c|}
\hline \multirow[t]{2}{*}{ Entry } & \multirow[t]{2}{*}{$\begin{array}{l}\text { Plant height } \\
(\mathrm{cm})\end{array}$} & \multirow[t]{2}{*}{$\begin{array}{l}\text { Biomass at } \\
50 \% \text { flow. } \\
(\mathrm{kg} / \mathrm{ha})\end{array}$} & \multirow[t]{2}{*}{$\begin{array}{l}1000 \text { grain } \\
\text { weight }(\mathrm{g})\end{array}$} & \multicolumn{2}{|l|}{ Days to } & \multicolumn{2}{|c|}{$\begin{array}{l}\text { Per days } \\
\text { production } \\
\text { kg/ha/day }\end{array}$} & \multicolumn{3}{|c|}{$\begin{array}{l}\text { Dry matter at } 50 \% \text { flow. } \\
\left(\mathrm{g} / \mathrm{m}^{2}\right)\end{array}$} & \multirow{2}{*}{$\begin{array}{l}\text { Stay green } \\
\text { at phy. } \\
\text { maturity (1- } \\
\text { 9 scale) }\end{array}$} \\
\hline & & & & $\begin{array}{l}50 \% \\
\text { flowering }\end{array}$ & \begin{tabular}{|l|} 
Phy. \\
Maturity
\end{tabular} & Grain & Fodder & \begin{tabular}{|l} 
Leave \\
$\mathrm{s}$ \\
\end{tabular} & Stem & $\begin{array}{l}\text { Panic } \\
\text { le }\end{array}$ & \\
\hline RSV1544 & 142 & 199 & 28 & 74 & 114 & 8.01 & 33.68 & 50 & 111 & 38 & $7-8$ \\
\hline RSV1572 & 152 & 207 & 27 & 75 & 115 & 6.29 & 35.07 & 49 & 120 & 38 & $5-6$ \\
\hline RSV1620 & 163 & 201 & 27 & 77 & 117 & 7.07 & 33.73 & 62 & 104 & 35 & $5-6$ \\
\hline RSV1640 & 161 & 244 & 27 & 75 & 115 & 6.36 & 20.61 & 50 & 164 & 30 & $5-6$ \\
\hline RSSV167 & 137 & 225 & 30 & 79 & 119 & 7.49 & 23.87 & 64 & 124 & 37 & $5-6$ \\
\hline CRS 49 & 135 & 225 & 32 & 74 & 114 & 7.58 & 31.51 & 44 & 146 & 35 & $5-6$ \\
\hline CRS 50 & 132 & 236 & 33 & 80 & 120 & 7.11 & 22.27 & 63 & 132 & 41 & $7-8$ \\
\hline PVR 930 & 135 & 190 & 28 & 75 & 115 & 7.90 & 17.56 & 52 & 102 & 36 & $7-8$ \\
\hline BRJ 229 & 141 & 197 & 31 & 80 & 120 & 7.41 & 19.08 & 53 & 112 & 33 & $7-8$ \\
\hline BRJ 343 & 164 & 200 & 28 & 80 & 120 & 6.89 & 21.88 & 61 & 101 & 38 & $5-6$ \\
\hline BRJ 235 & 138 & 287 & 25 & 78 & 118 & 7.75 & 32.73 & 63 & 184 & 41 & $5-6$ \\
\hline BRJ 376 & 103 & 276 & 26 & 81 & 121 & 7.26 & 22.16 & 68 & 168 & 40 & $7-8$ \\
\hline BRJ 341 & 139 & 228 & 34 & 79 & 119 & 3.65 & 19.98 & 63 & 119 & 46 & $5-6$ \\
\hline M 35-1(C) & 152 & 184 & 29 & 79 & 119 & 5.43 & 26.29 & 51 & 102 & 31 & $7-8$ \\
\hline P. Suchitra (C) & 151 & 241 & 34 & 80 & 120 & 5.78 & 27.40 & 75 & 122 & 45 & $5-6$ \\
\hline $\begin{array}{l}\text { P. Anuradha } \\
\text { (C) }\end{array}$ & 120 & 197 & 29 & 74 & 108 & 8.20 & 22.70 & 46 & 113 & 38 & $5-6$ \\
\hline Correlation & +0.48 & +0.29 & +0.69 & +0.09 & +0.33 & +0.67 & +0.582 & +0.36 & +0.639 & +0.24 & - \\
\hline
\end{tabular}


For the final yield genotype RSV 1640 gave $3374 \mathrm{~kg} / \mathrm{ha}$ grain yield and $5054 \mathrm{~kg} / \mathrm{ha}$ fodder yield on medium soil. While Phule Anuradha gave highest grain yield $935 \mathrm{~kg} / \mathrm{ha}$ and 2588 $\mathrm{kg} / \mathrm{ha}$ fodder yield on shallow soil. Chavan et $a l$. , (2012) studied the seven rabi genotypes and reported that the grain yield ranged from 17.3 to $26.8 \mathrm{q} / \mathrm{ha}$ on medium soil. Similar results are reported by Shinde and Narkhede (1998).

The grain yield was positively correlated with biomass at harvest, HI \%, 1000 grain weight, RLWC \%, stay green at physiological maturity, while per day production of grain and fodder yield negatively correlated with leaf temperature differences. On medium soil genotype RSSV 1640 was found significantly superior over the checks. On shallow soil, none of the genotype was found significantly superior over the check Phule Anuradha.

For medium soil new Rabi sorghum genotype RSV 1640 is the best one while for shallow soil Phule Anuradha is the best Rabi sorghum genotype which gives higher grain and fodder yield on the basis of the physiological parameters.

\section{References}

AOAC, (1990). Official Methods of Analysis. $15^{\text {th }}$ Edn. Association of Official Analytical Chemists, Washington, DC, pp.113-127.

Barrs, H. D. and Weatherly, P. E. (1962). Physiological indices for high yield potential in wheat. Indian J. of Plant Physiol. 25: 352-357.

Chavan U. D., Pansare, S. S., Patil, J. V. and Shinde, M. S. (2015). Preparation and Nutritional Quality of Sorghum Papads. Int. J. Curr. Microbiol. App. Sci. 4(5): 806-823.

Chavan, J. K., Salunkhe, D. K. (1984). Structure of sorghum grain in nutritional and processing quality of Sorghum. Qual.
Plant. Pl. Foods Human Nutr 29: pp.2131

Chavan, J.K., Chavan, U.D. and Nagarkar, V.D. (1989). Effects of malting and fermentation on nutritional quality of sorghum. J. Maharashtra Agric. Univ., 14 (2) : 246-247.

Chavan, U. D., Patil, J. V. (2010). Grain Processing of Sorghum. Ibdc publishers, Lucknow. pp. 10-15

Chavan, U. D., Patil, J. V. and Shinde M. S. (2009). Nutritional and roti quality of sorghum genotypes. Indonesian Journal of Agriculture Science 10: 80-87.

Chavan, U. D., Patil, J. V. and Shinde, M. S. (2012). Nutritional quality of high yielding rabi sorghum genotypes. J. Agric. Res. Technol. 37: 46-50.

Chavan, U.D., Bhagwat, V.R., Ratnavati, C.V., Patil, J.V., Gawali, H.S and Shailaja, V. (2010). Jwariche Ruchakar Padartha. Sorghum Research Centre, Rajendranagar, Hyderabad (India).pp. 26

Chavan, U.D., Chavan, J.K. and Kadam, S.S. (1988). Effect of fermentation on soluble proteins and in-vitro protein digestibility of sorghum, green gram and sorghum + green gram blends. J. Food Sci. 53 : 15741575.

Donald, C. M. (1962). In earch of yield. J. of Australian Institute of Agric. Sci. 28: 171-178.

Hiremath, S. M. and Parvatikar, S. R. (1985). Growth and yield analysis in sorghum. Identification of genotypes with low leaf area and high dry matter production. Sorghum News Letter. 28: 108-109.

Iwata, F. (1984). In Physiological aspect of dry farming (ed. U. S. Gupta), Oxford and IBH Publ. Co., New Delhi. Pp. 351-370.

Klages, K. H. W. (1958). Ecological crop geography, pp. 100-103.

Kulkarni, L. P., Narayana, R. and Krishna Sastry, K. S. (1981). Photosynthetic efficiency and translocation in relation to leaf characters and productivity in sorghum genotypes. Sorghum Newsletter. 24: 124-125. 
Kusalkar, D. V., Awari, V. R., Pawar, V. y. and Shinde, M. S. (2003). Physiological parameters in relation to grain yield in Rabi sorghum on medium soil. Adv. Plant Sci. 16: 119-122.

Michniewicz, J., Biliaderis, C. G. and Bushuk, W. (1991). Effect of added pentosans on some physical and technological characteristics of dough and gluten. Cereal Chemistry 68: 252-258.

Murty D. S. and Subramanian V. (1981). Sorghum Roti: I. Traditional Methods of Consumption and Standard Procedures for Evaluation. Proceedings of the International Symposium on Sorghum Grain Quality: 73-78.

Nandini C. D. and Salimath, P. V. (2001). Structural features of arabinoxylans from sorghum having good roti-making quality. Food Chemistry 74: 417 - 422.

Narkhede, B. N., Shinde, M. S. and Patil, S. P. (1998). Association of physiological parameters with grain yield of Rabi sorghum. Ann. Pl. Physiol. 12: 65-66.

Nirmal, S. V. and Patil, J. V. (2008). A new drought tolerant genotype of Rabi sorghum SPV 1546 (Phule Chitra). Ann. Pl. Physiol. 22: 165-168.

Nirmal, S. V., Dalvi, U. S., and Gadakh, S. R. (2013). Effect of water stress condition on root characteristics of sorghum plant under irrigated and rainfed conditions. Crop res. 45: 132-135.

Panse, V. G. and Sukhatme, P. V. (1985). Statistical Methods for Agricultural Workers $2^{\text {nd }}$ Edn. I.C.A.R., New Delhi.

Rajput, R. P. (1980). Response of soybean crop to climate and soil environments. Ph. D. Thesis, IARI, New Delhi.
Rao Prasada, K. E. and Murty, D. S. (1981). Sorghum for Special Uses. Proceedings of the International Symposium on Sorghum Grain Quality: 129-134.

Shinde, M. S. and Narkhede, B. N. (1998). Association of physiological parametrs with grain yield of Rabi sorghum. Ann. Plant Physiol. 12: 65-66.

Shobha, V., Kasturiba, B., Naik, R. K. and Yenagi, N. (2008). Nutritive Value and Quality Characteristics of Sorghum Genotypes. Karnataka Journal of Agriculture Science 20: 586-588

Stickler, F. C., Wearden, S. and Pauli, A. W. (1961). Leaf area determination in grain sorghum. Agron. J. 53: 178-188.

Subramanian, V. and Jambhunathan, R. (1982). Properties of sorghum grain and their relationship to rot quality. In: International Symposium on sorghum grain quality. ICRISAT, Patancheru, India. pp. 280-288.

Subramanian, V. and Jambunathan, R. (1981). Properties of Sorghum Grain and their Relationship to Roti Quality. Proceedings of the International Symposium on Sorghum Grain Quality: 286-288.

Vietor, R. J., Angelino, S. A. G. F. and Voragen, A. G. J. (1992). Structural features of arabinoxylans from barley and malt cell wall material. Journal of Cereal Science 15: 213-222.

Watson, D. J. (1947). Comparative physiological studies on growth of field crops. I. variation in net assimilation rate and leaf area between species and varieties and within years. Ann. Bot. 11: 41-76.

\section{How to cite this article:}

Chavan, U.D., S.V. Nirmal, S.R. Gadakh, M.S. Shinde, V.R. Patil, V.R. Awari and Dalvi, U.S. 2017. Physiological Evaluation of Genotypes for Drought Tolerance on Receding Soil Moisture. Int.J.Curr.Microbiol.App.Sci. 6(6): 2174-2183. doi: https://doi.org/10.20546/ijcmas.2017.606.256 